\title{
Single-shot positron annihilation lifetime spectroscopy with LYSO scintillators
}

\author{
A. M. Alonso, B. S. Cooper, A. Deller, D. B. Cassidy \\ Department of Physics and Astronomy, University College London, Gower Street, London, WC1E 6BT, UK
}

\begin{abstract}
We have evaluated the application of a lutetium yttrium oxyorthosilicate (LYSO) based detector to single-shot positron annihilation lifetime spectroscopy. We compare this detector directly with a similarly configured $\mathrm{PbWO}_{4}$ scintillator, which is the usual choice for such measurements. We find that the signal to noise ratio obtained using LYSO is around three times higher than that obtained using $\mathrm{PbWO}_{4}$ for measurements of Ps excited to longer-lived (Rydberg) levels, or when they are ionized soon after production. This is due to the much higher light output for LYSO (75\% and $1 \%$ of $\mathrm{NaI}$ for LYSO and $\mathrm{PbWO}_{4}$ respectively). We conclude that LYSO is an ideal scintillator for single-shot measurements of positronium production and excitation performed using a low-intensity pulsed positron beam.
\end{abstract}

Keywords: LYSO; Positronium; Annihilation; Spectroscopy; Rydberg

\section{Introduction}

The development of positron trapping and manipulation techniques [1] has made it possible to produce pulsed positrons from a standard DC positron beam [2]. When a pulsed positron beam is used to generate a positronium (Ps) gas, a burst of annihilation gamma radiation is created. The time and energy characteristics of these photons contain information regarding Ps formation and annihilation processes, and observations thereof can be used to detect Ps-Ps scattering [3], Ps-surface effects in solid-state materials [4 or laser-induced atomic transitions [5-7].

Conventional gamma-ray detectors are usually designed to observe single events and cannot process many thousands of photons arriving in a few hundred nanoseconds. Thus, in order to use such detectors with an intense pulsed beam one has to severely limit its acceptance, losing almost all of the available information [6]. Solutions to this problem were first suggested by Mills Jr. 8, and were eventually implemented in a technique called single-shot positron annihilation lifetime spectroscopy (SSPALS) 9. In this approach an appropriate gamma-ray detector is directly connected to a fast oscilloscope, so that the gamma-ray flux following an intense positron pulse [10] is monitored as a function of time. The detectors that have been used for this are scintillators or Cherenkov radiators optically coupled to a photomultiplier tube (PMT) [11].

This technique was originally developed for studies of Ps-Ps interactions [3, which happen on a short time scale and require intense, high-density, positron pulses [10. The optimal detector for such measurements is one with a decay constant that is short compared to the 142 ns groundstate Ps lifetime 12, and which has a relatively low light output in order to mitigate saturation of the PMT. Although $\mathrm{PbF}_{2}$ [1] and heated $\mathrm{BaF}_{2}$ [13 were shown to be viable options partially fulfilling these criteria, lead tungstate $\left(\mathrm{PbWO}_{4}\right.$, often abbreviated to $\left.\mathrm{PWO}\right)$ proved to have the best overall performance of the materials studied. This technique has made it possible to perform a wide variety of measurements involving Ps produced at both high (e.g., 14, 15]) and low (e.g., [16, 17]) densities.

Recent work has focused on the production and study of Rydberg Ps atoms using low-intensity, low-density, positron pulses 18 20. Since these atoms are generally long lived [21, the timing constraints on an appropriate SSPALS detector may be relaxed for Rydberg studies. The $\sim 12$ ns decay time of PWO is well-suited for the study of short-lived effects since it allows one to integrate lifetime spectra almost from the moment of Ps atom creation. With long-lived Rydberg states, however, the annihilation events of interest may occur on completely different timescales, and the regions of interest in the corresponding lifetime spectra will change accordingly [22]. Therefore, for measurements of this kind it can be advantageous to use a slower scintillator with a higher light output.

We have tested this possibility using Cerium doped lutetium yttrium oxyorthosilicate, $\left(\mathrm{Lu}_{2(1-x)} \mathrm{Y}_{2 x} \mathrm{SiO}_{5}\right.$ :Ce). LYSO 23] (as well as its predecessor LSO 24]) has a useful combination of properties, namely its short radiation length $(\sim 1 \mathrm{~cm})$, high density $\left(8 \mathrm{~g} \mathrm{~cm}^{-3}\right)$, high light output ( $\sim 75 \%$ of NaI), and relatively fast decay time (40 ns). As a result these materials have found applications in areas such as positron emission tomography (PET) 25] and high energy physics [26. Because of its high stopping power LYSO is well suited for studies of low-energy photons [27, which is useful not only in PET scanners 28 but also other positron physics applications (e.g., 29 31). As we shall discuss, these same properties also make LYSO a good material to use for SSPALS, especially when conducting 
experiments with Rydberg Ps atoms.

\section{Experimental arrangement}

SSPALS measurements are performed using a pulsed positron source. In the present case we generate a lowdensity Ps gas by implanting around $10^{5}$ positrons in a $5 \mathrm{~ns}$ wide pulse into a mesoporous silica film 22 . These materials emit Ps atoms into vacuum following positron bombardment with an efficiency of around 25\% 32. Groundstate Ps atoms are excited to higher lying levels using a pair of Nd:YAG pumped ns pulsed dye lasers: one broadband $(\sim 100 \mathrm{GHz}) \mathrm{UV}$ laser $(\lambda \sim 243 \mathrm{~nm})$ and one narrow band $(\sim 5 \mathrm{GHz})$ IR laser $(\lambda \sim 750 \mathrm{~nm})$. Rydberg atoms are produced by a two-step transition via $n=2$, as described elsewhere [6, 20].

The detector used was a $10 \times 10$ array of LYSO crystals, each of which was $4 \times 4 \times 20 \mathrm{~mm}$. The individual crystals were wrapped on the long sides with reflective Teflon tape, and the resulting $40 \times 40 \times 20 \mathrm{~mm}$ assembly was optically coupled to a photomultiplier tube (PMT) via a $150 \mathrm{~mm}$ long light guide. The light guide was an acrylic cylinder with a $50 \mathrm{~mm}$ diameter wrapped in reflective Teflon tape, and was necessary to minimize the effect on the PMT of the $\sim 100 \mathrm{G}$ magnetic field used to transport the positrons. For comparison we also used an $50 \times 38 \times 25 \mathrm{~mm}$ PWO crystal coupled to a PMT with a similar light guide.

The detector positions relative to the Ps production and excitation region are indicated in Fig. 1 and were arranged so that the solid angles subtended by each detector relative to the $\mathrm{Ps}$ production region were approximately equal. All of the data presented here were recorded simultaneously from the two detectors, so that the positron and positronium conditions were identical. The PMT's used in each case were different. The LYSO detector was attached to an EMI type 9954KA PMT operated with a supply voltage of $-0.9 \mathrm{kV}$. The PWO was attached to a Hamamatsu H10570 PMT, operated with a supply voltage of $-1.4 \mathrm{kV}$. These supply voltages were chosen to avoid PMT saturation.

One potential disadvantage of LYSO is that about $2.6 \%$ of naturally occurring $\mathrm{Lu}$ is the isotope ${ }^{176} \mathrm{Lu}$, which is radioactive and has a half-life of $10^{10}$ years. The decay of this isotope and daughter products lead to the emission of beta particles, and gamma rays with energies of 89,202 , and $307 \mathrm{keV}$ 24. This can result in an unwanted background signal in PET scanners (for example), but is not important in single shot measurements since the probability of an event occurring in the $\sim 1 \mu \mathrm{S}$ time windows typically used in SSPALS measurements is negligible.

LYSO is available commercially from a variety of vendors, with only slight variations in their properties 33]; the crystals used in this work are of unknown provenance as they were obtained via the secondary market, from an on-line reseller. The crystal dimensions are consistent with those used in PET scanners (e.g., 34]).

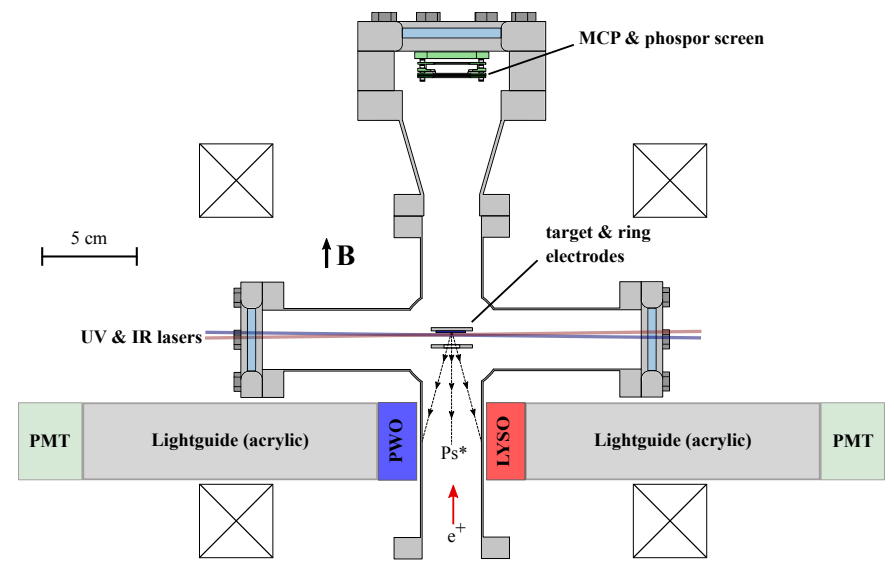

Figure 1: Schematic layout of the target chamber and gamma-ray detectors used in this work. A magnetic field of around $100 \mathrm{G}$ is produced in the Ps excitation region by a pair of coils. An electrode placed in front of the mesoporous silica film allows the electric field in the excitation region to be controlled without affecting the positron beam implantation energy.

\section{Ground-state positronium}

The SSPALS technique involves directly recording the output of a gamma-ray detector with an oscilloscope 9 . The gamma-ray signals obtained in this way are in effect lifetime spectra, examples of which obtained using LYSO and PWO detectors are shown in Fig. 2. These data were recorded with the positron beam implanted into a mesoporous silica film $\left(\mathrm{SiO}_{2}\right)$, from which $\mathrm{Ps}$ is expected to be emitted, and into a piece of untreated copper (the electrode in front of the silica film), from which we expect almost no Ps to be emitted. The formation of Ps is readily apparent in both detectors as a surplus of counts at later times after the initial peak. This "prompt" peak is due to positrons that annihilate rapidly, either in direct electron-positron interactions following implantation, or via the formation of short-lived (125 ps) singlet Ps atoms [12]. The width of the peak is determined by the width of the incident positron beam and/or the time response of the detector used. Here the positron pulse width is around 5 ns FWHM, and so the observed peak widths are mostly due to the properties of the LYSO and PWO scintillators.

Single-shot lifetime spectra are typically analyzed using the parameter $f_{\mathrm{d}}$, defined as

$$
f_{\mathrm{d}}=\int_{B}^{C} V(t) d t / \int_{A}^{C} V(t) d t .
$$

The integration regions given by $A, B$ and $C$ are selected according to the type of detector used, and also the processes to be studied 22 . The amount of Ps formed is related to $f_{\mathrm{d}}$ (see [35]) but numerous factors must be accounted for before a direct conversion to the actual Ps fraction can be extracted. The spectra in Fig. 2 have been analyzed using $B=3 \times \tau$, where $\tau$ is the scintillator decay constant (see $f_{\mathrm{d}}$ values in Fig. 2). This makes it likely that a significant fraction of the integrated spectrum will 


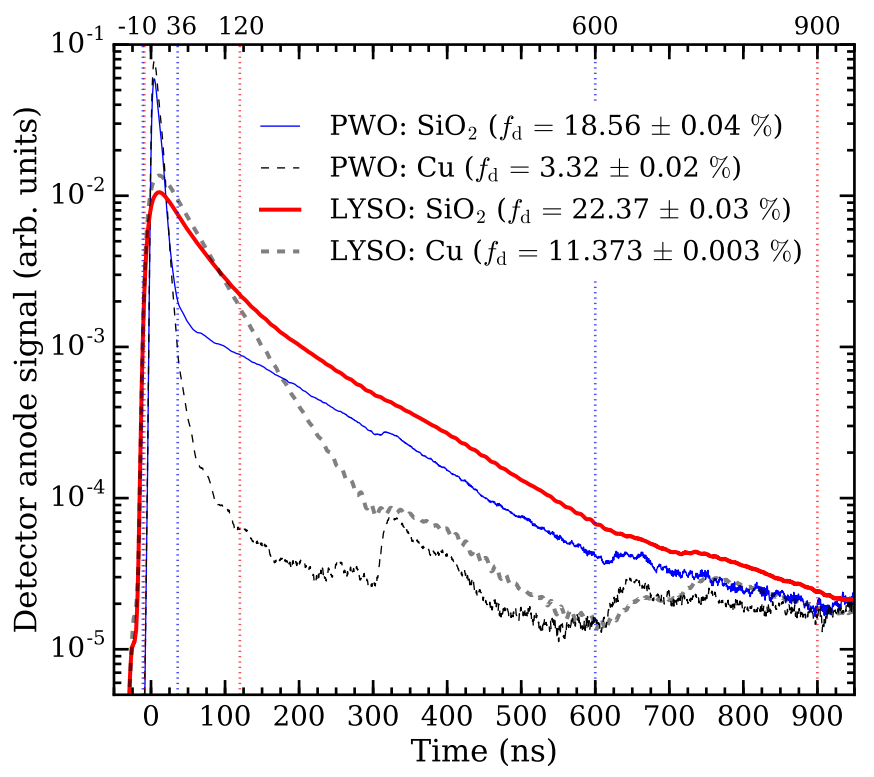

Figure 2: SSPALS lifetime spectra recorded with PWO and LYSO detectors, with positrons implanted into a mesoporous silica film or a piece of untreated $\mathrm{Cu}$, as indicated in the legend. These spectra are the average of 860 individual shots and were acquired in approximately 15 minutes. The quoted $f_{\mathrm{d}}$ values were calculated using the indicated time windows as described in the text and represented by the color-coded vertical dashed lines and the corresponding labels above, (i.e., for PWO the boundaries $A, B$ and $C$ were $-10,36$, and 600 ns respectively and for LYSO the boundaries $A, B$ and $C$ were $-10,120$, and 900 ns respectively).

be due to photons originating from long-lived Ps annihilation, as opposed to light from the prompt peak delayed by the scintillator decay. The actual Ps fraction from mesoporous silica films nominally identical to those used here is around $25 \% 36$.

Figure 3 shows $f_{\mathrm{d}}$ obtained from the data of Fig. 2 using different values of of $B$. These data indicate that if $B$ is too close to the prompt peak events that are not related to Ps formation are included in the signal, giving a higher $f_{\mathrm{d}}$. Conversely, if $B$ is too far away from the peak the signal will miss events that are caused by Ps annihilation, giving a lower $f_{\mathrm{d}}$. For PWO there is a clear increase in the smoothly varying $f_{\mathrm{d}}$ when $B$ is less than around $30 \mathrm{~ns}$, indicating that this is the point at which peak events start to be included.

For LYSO the time at which the faster $f_{\mathrm{d}}$ increase occurs is less obvious, but is in the region of $B \sim 150 \mathrm{~ns}$. Thus, the optimal region of integration for this detector excludes the signal due to Ps decay for around one lifetime, which is the primary reason why PWO was initially selected for SSPALS experiments [11. These data demonstrate that $f_{\mathrm{d}}$ cannot be interpreted directly as the Ps fraction, since one can never integrate all the way to zero time. Moreover, there are other factors that must be included, such as background signals and the efficiency with different photon energies are detected [35].

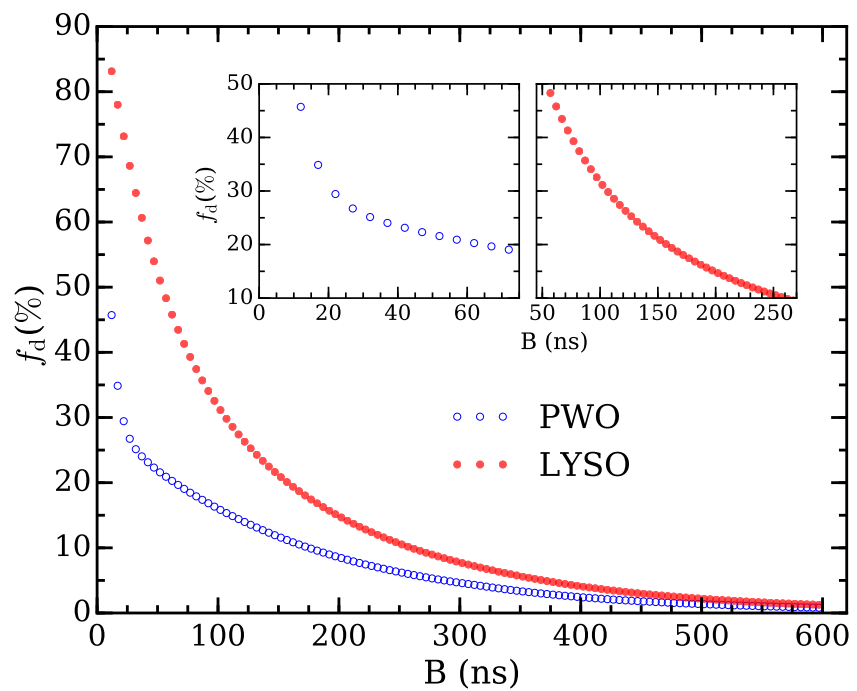

Figure $3: f_{\mathrm{d}}$ obtained using different values of $B$ for LYSO and PWO detectors. The values of $A$ and $C$ were -10 ns and 600 ns respectively for PWO, and $-10 \mathrm{~ns}$ and $900 \mathrm{~ns}$ for the LYSO detector. The two insets show the region where peak events stop being included in $f_{\mathrm{d}}$ as explained in the text.

\section{Excited-state positronium}

Changes in lifetime spectra due to mechanisms that affect Ps decay rates are characterized by the signal parameter $S_{\gamma}$, given by

$$
S_{\gamma}=\left(f_{\mathrm{bk}}-f_{\mathrm{sig}}\right) / f_{\mathrm{bk}},
$$

where $f_{\mathrm{bk}}$ refers to the background Ps signal, which is the value of $f_{\mathrm{d}}$ obtained without the perturbing influence being studied, and $f_{\text {sig }}$ is the value obtained when it is present. In the present work $f_{\text {sig }}$ refers to the presence of both the UV and IR lasers tuned to the relevant frequency to excite Ps into Rydberg states, and $f_{\mathrm{bk}}$ refers to the case where the IR laser is tuned to be off resonance.

There are several processes that may affect Ps decay rates, such as spin exchange quenching or $\mathrm{Ps}_{2}$ molecule formation 14, the application of a strong magnetic field 37, optical excitation and/or photoionization [16] or Ps quenching with paramagnetic centers 38 . When studying such effects using $S_{\gamma}$ as the metric it is necessary to adjust the integration parameters ( $c f$ Eq. 1) according to the relevant time scales. That is, the optimal analysis will differ depending on whether the Ps atoms are being made to annihilate at a rate that is faster or slower than the unperturbed vacuum rate.

\subsection{Short-lived Ps}

If Ps atoms are excited to $n=2$ levels in a magnetic field, Zeeman mixing between singlet and triplet sub-levels can cause long-lived Ps atoms to decay at a faster rate in a process known as magnetic quenching [39]. If electric fields are also present this process can be enhanced by 

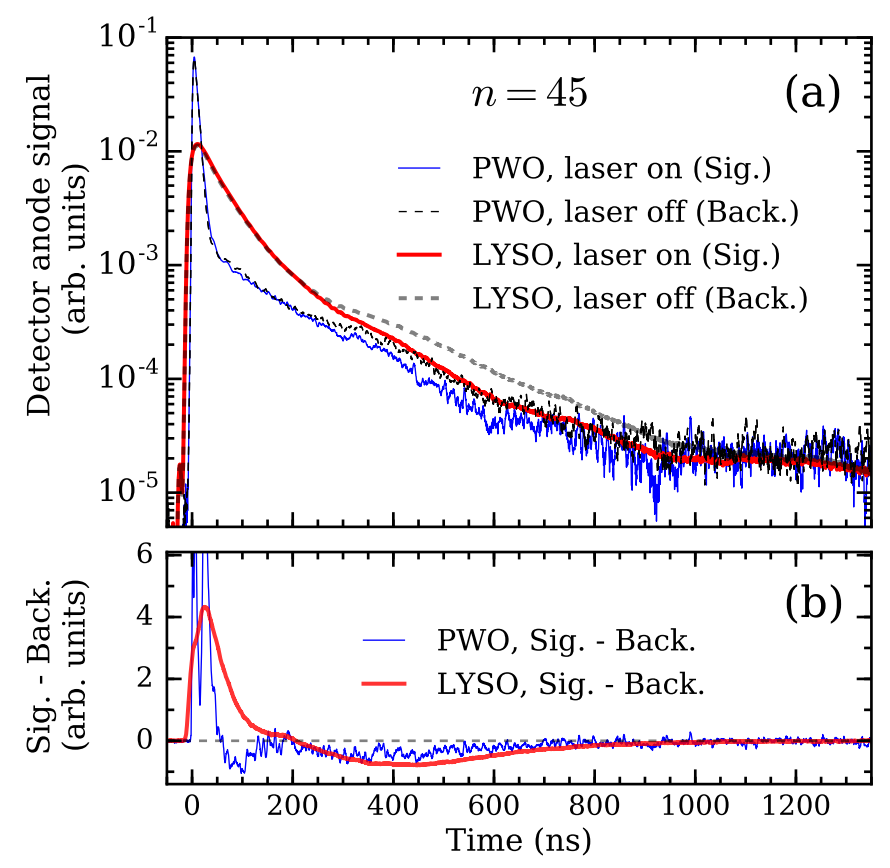

Figure 4: (a) Single-shot lifetime spectra from LYSO and PWO detectors with the IR laser tuned to excite $n=2$ Ps to $n=45$ $(\lambda=730.45 \mathrm{~nm})$ and $(\mathrm{b})$ the difference between the laser on and off spectra shown in (a). The spectra are averages of 275 individual shots and are all normalized to the area between $-10 \mathrm{~ns}$ and $1200 \mathrm{~ns}$. Note that in (b) the PWO early peaks are truncated to better illustrate the difference in the statistics between the two data sets. The amplitudes of the first and second peaks are 6 and 10 respectively.

Stark mixing [40. Similarly, if Ps atoms are optically excited they may subsequently be ionized, either optically [16, or in the case of highly excited (Rydberg) states, by electric fields [20]. SSPALS can detect such events; for rapid annihilation they will appear as an increase in the gamma-ray signal in the prompt peak, and a decrease in the delayed photon flux. That is, $f_{\mathrm{d}}$ will decrease, and $S_{\gamma}$ will have a positive value.

Figure 4 shows lifetime spectra measured with both a LYSO and a PWO detector, obtained with the IR laser tuned to excite $n=2$ atoms to the $n=45$ level. These atoms (which we cannot resolve spectroscopically [20]) are field ionized in an electric field of $\sim 100 \mathrm{~V} \mathrm{~cm}^{-1}$. The increased annihilation due to this ionization can be observed most clearly in the difference spectra, shown for both detectors in Fig. 4 (b). The initial signal (observed for times $\leq 50 \mathrm{~ns}$ ) is due to laser induced annihilation events, while the later negative dip peaking at around $400 \mathrm{~ns}$ is due to the absence of annihilation events. Similar profiles are obtained if the $n=2$ Ps atoms are directly ionized using IR light at $729 \mathrm{~nm}$.

The two peaks (also seen with direct ionization) are due to positrons that do not immediately return to the target and annihilate. These cannot be directly resolved with the LYSO detector, although there is a visible shoulder in the difference curve. This illustrates the fact that there may be some measurements for which the superior time
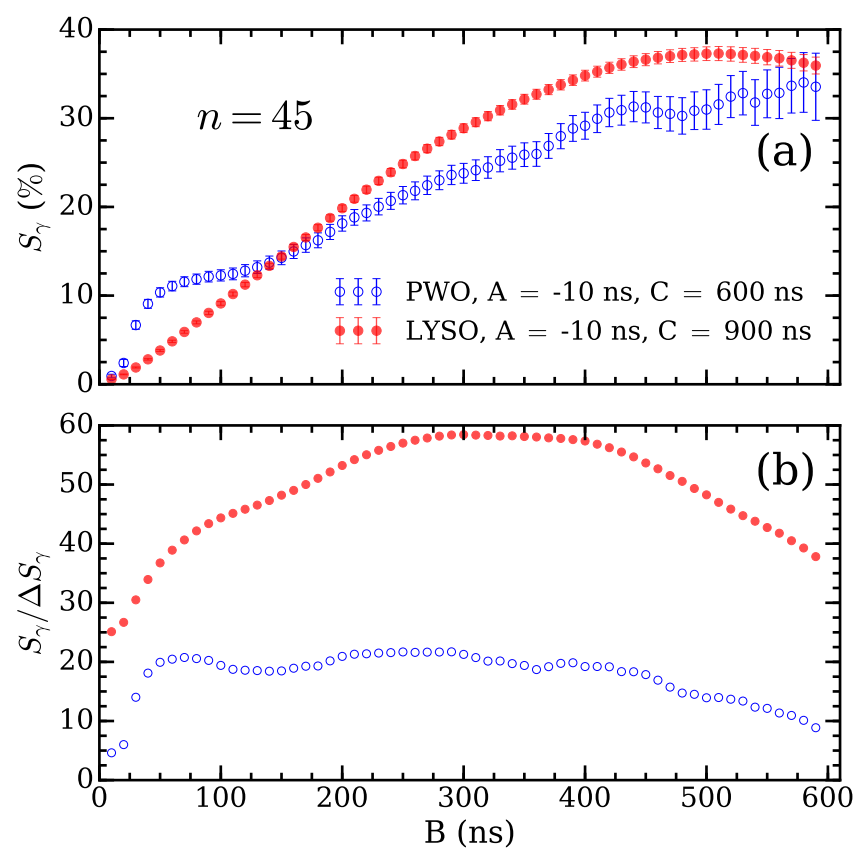

Figure 5: (a) The signal $S_{\gamma}$ and the SNR (b) obtained from the data shown in Fig. 4 as a function of the integration bound $B$.

resolution of PWO might make it a better choice, despite the reduced statistics.

Data of the type shown in Fig. 4 can be analyzed to obtain $S_{\gamma}$ values by selecting the most appropriate values of the time windows used to generate the $f_{\mathrm{d}}$ values (see Eq. 1). That is, just as the measurement of the amount of ground-state Ps present (i.e., $f_{\mathrm{d}}$ ) can be optimized by choosing $B$ appropriately (see Fig. 3), so too is $S_{\gamma}$ highly dependent on the choice of $B$. In this case, however, one has to select $B$ based on the specific process being measured. In general the values for $A$ and $C$ are fixed by the interval over which annihilation radiation can be detected, although one can partially reduce the amount of noise in the signal by restricting $C$.

Figure 6 shows $S_{\gamma}$ and the signal-to-noise ratio (SNR) obtained from the data shown in Fig. 4 as a function of $B$. It is evident from these data that there is an optimal integration region that enhances not only the magnitude of the signal but, more importantly, the SNR.

\subsection{Long-lived Ps}

If Ps atoms are excited to Rydberg states there will be virtually no annihilation [40] and their lifetimes will be determined by their radiative lifetimes or other interactions. In the present experiment some Ps atoms in Rydberg states are able to collide with the vacuum chamber walls (see Fig. 1), which provides an annihilation signal regardless of the atomic state. This signal is, however, quite different from that of short-lived Ps atoms. Figure 7 shows lifetime spectra measured with both a LYSO and a PWO detector, obtained with the lasers tuned to excite 


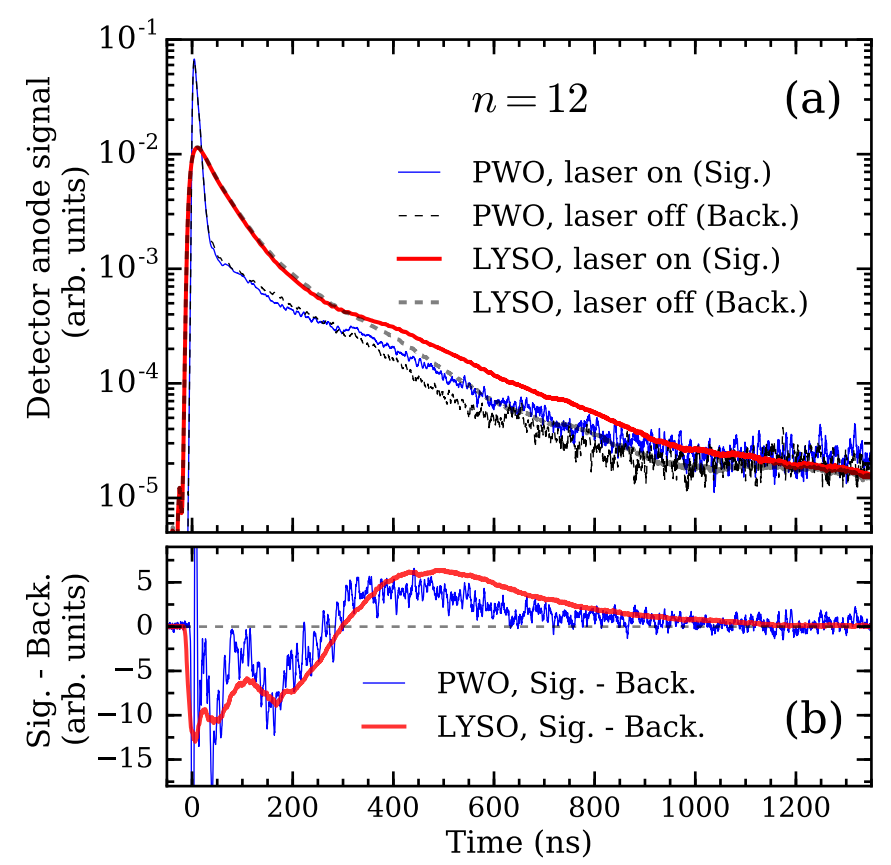

Figure 6: (a) Lifetime spectra recorded simultaneously with LYSO and PWO detectors with the IR laser tuned to excite $n=2$ Ps to $n=12(\lambda=749.84 \mathrm{~nm})$ and (b) the difference between the laser on and off spectra shown in (a). The spectra are averages of 400 individual shots and are all area normalized.

$n=12$ Rydberg states. The mean radiative lifetimes of these atoms depends on exactly which states are generated, which is not precisely defined due to the slightly inhomogeneous fields present in the excitation region. However, it is likely to be in excess of $1 \mu \mathrm{S} 41$. Since the mean Ps speed is of the order of $10^{5} \mathrm{~m} \mathrm{~s}^{-1}$ [17], the $\sim 5 \mathrm{~cm}$ flightpath to the chamber walls will occur with essentially no losses to radiative decay.

The data shown in Fig. 7 are significantly different to those of Fig. 4. When longer-lived atoms are generated, the laser-on curves exhibit an increase in the gamma-ray signal at later times rather than a deficit. Accordingly, the difference curves are inverted, with a dip at early times indicating fewer annihilations, and a peak at later times due to increased annihilations following wall collisions. There is also a small peak evident at around $100 \mathrm{~ns}$, which is due to Ps atoms annihilating on the ring electrode located around $8 \mathrm{~mm}$ from the Ps target (see Fig. 11. The apparent shift in time of the LYSO difference curve relative to the PWO is due to the different time response of the detector, and also the fact that the LYSO is able to detect later events more efficiently than the PWO.

Figure 8 shows $S_{\gamma}$ and the SNR obtained from the data shown in Fig. 7 as a function of $B$. This is the same analysis used to generate Fig. 6 but in this case a negative signal is obtained, since the delayed annihilations are increased by the laser excitation (see section 3). These data show that maximizing $S_{\gamma}$ does not in general optimize the SNR.
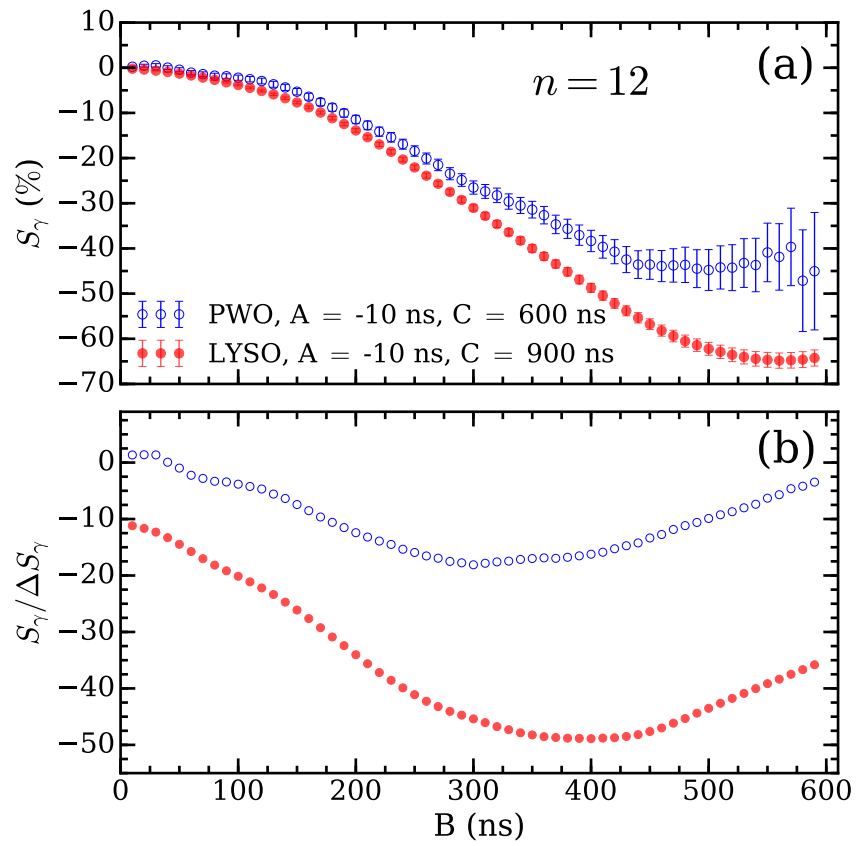

Figure 7: (a) The signal $S_{\gamma}$ and the SNR (b) obtained from the data shown in Fig. 7 as a function of the integration bound $B$.

\section{Applications}

The primary result of our investigation is that the high light output from LYSO offers a significant improvement over PWO in terms of the SNR, even when Ps is probed at early times. This is demonstrated explicitly in Fig. 9 . which shows the SNR as a function of the data acquisition time. These data are obtained using optimal time windows. The LYSO SNR is around a factor of 3 higher than the PWO SNR, indicating that the data acquisition time could be reduced by almost an order of magnitude without degrading the statistics. The statistical limitations in any measurement will also be affected by other noise sources, such as electronic noise from the PMT, RF pick-up from the high voltage buncher, digitization noise from the oscilloscope, and so on. However, these will be mostly the same regardless of the type of scintillator used.

Increasing the efficiency with which SSPALS data can be collected is very desirable because, even when using a positron trap [1, the relatively low number of positrons available is always an experimental limitation [42]. As a result it is not uncommon in positronium-laser experiments for data acquisition to take days or weeks. This situation sets some practical limits on what can be achieved in this area. For example, measurements using delicate surfaces may be significantly more difficult if the surface conditions are not stable on the time scale of the data acquisition (e.g., [38, 43]). On the other hand, in experiments that are not limited in this way, the possibility of obtaining higher quality data remains attractive.

As may be seen in Fig. 6 and 8 , the magnitude of the signal parameter $S_{\gamma}$ depends on the time windows selected. 


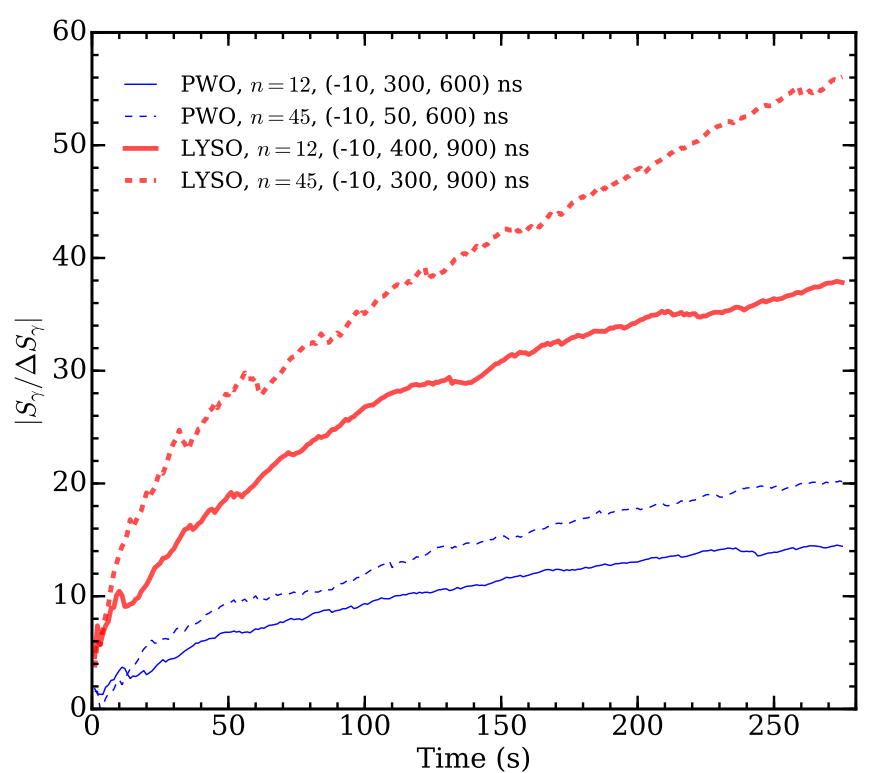

Figure 8: The SNR for LYSO and PWO detectors as a function of the data acquisition time. Data are shown for the two cases considered in section 4 namely field ionization of $n=45$ Ps, and delayed collisions of $n=12 \mathrm{Ps}$ with the chamber wall. The acquisition rate was $1 \mathrm{~Hz}$ The values of $A, B$ and $C$ used in the analysis are given in the legend for each case in the format $(A, B, C)$.

It also differs in the two types of measurements performed ( $n=45$ and $n=12$ ) because the solid angle subtended by the detectors are different, making them less sensitive to annihilations occurring at earlier times. Nevertheless, $S_{\gamma}$ can be used to monitor relative changes in excitedstate populations, such as may be obtained in laser spectroscopy.

Figure 10 shows the lineshape of transitions between $n=2$ and $n=12$. The $n=2$ atoms are excited via a $1^{3} \mathrm{~S}_{1} \rightarrow 2^{3} \mathrm{P}_{1}$ transition which produces mixed $n=2$ states 40. However the structure of the $n=2$ sub-levels is not apparent in the measured transitions to $n=12$ due to the limited spectral resolution of our experiment 22 . The observed line is essentially featureless, therefore, but is broadened due to the $\sim 100 \mathrm{~V} \mathrm{~cm}^{-1}$ electric field in the excitation region and slightly shifted from the resonance wavelength due to a slight angle of the excitation lasers with the direction of travel of the Ps atoms. The improvement in the data quality using LYSO is clear, and is fully consistent with the data shown in Fig. 9.

If the electric field in the Ps excitation region is increased, lineshapes will be Stark broadened to a much greater extent than is shown in Fig. 10. If the field is high enough the individual Stark states may become visible, provided the probe laser resolution is sufficient [20]. However, by splitting the line in this way the available signal is concomitantly reduced, making these Stark states difficult to observe. Figure 11 shows a measurement of this type. Ps atoms excited to $n=11$ in an electric field of $\sim 1.875 \mathrm{kV} \mathrm{cm}^{-1}$ are sufficiently broadened to allow the

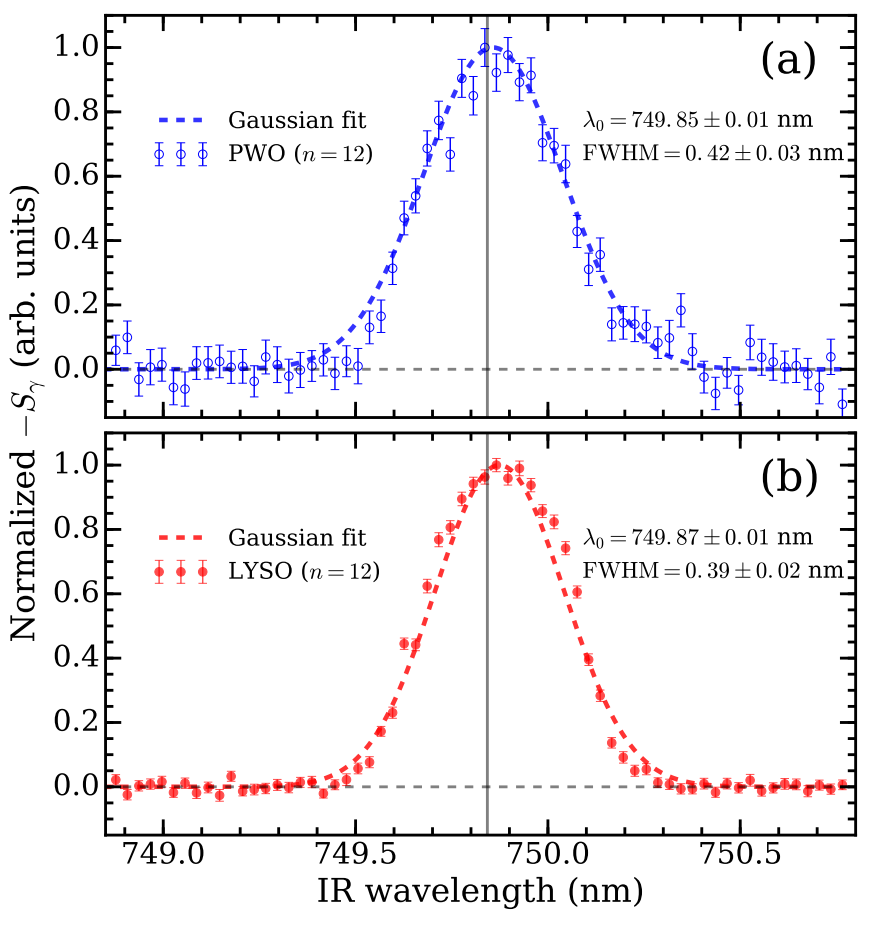

Figure 9: Lineshape for $n=12$ Ps excitation, recorded with LYSO and PWO detectors. The acquisition time was 400 seconds per point, or approximately 7 hours for the entire spectra. These data have been normalized to the maximum peak amplitude. The vertical line represents the wavelength of the transition from the $n=2$ gross energy level as described by the Bohr model.

individual Stark states to be resolved. This measurement was done using $n=11$ so that a strong field could be applied without causing neighboring $n$ states to overlap, which is just starting to occur at the shorter wavelengths.

Again the improved statistics obtained using LYSO are evident. In this measurement there may be additional effects occurring, since different Stark states could have their trajectories altered differently after excitation, leading to a state-selective detection. The possibility of observing such effects in a reproducible manner will be the subject of future work; the aim is to produce an electrostatic Ps lens 44. Being able to see small effects caused by small shifts of Ps trajectories will help to optimize such arrangements, which are extremely challenging to implement owing to the large velocity distributions typically associated with efficient Ps production.

The production of Ps atoms in selected Stark states is expected to be useful as it will facilitate the manipulation of these atoms via their large dipole moments (e.g., [45]). The ability to control the translational motion of Ps atoms in this way, or even to decelerate and trap them [46], would open up many new experimental possibilities. For example, performing a free-fall Ps gravity measurement has been discussed for some time [47. but requires a slow and focused beam of Ps atoms. Similarly, the availability of cold Ps would mitigate second order Doppler effects that are detrimental to precision spectroscopy [48. 


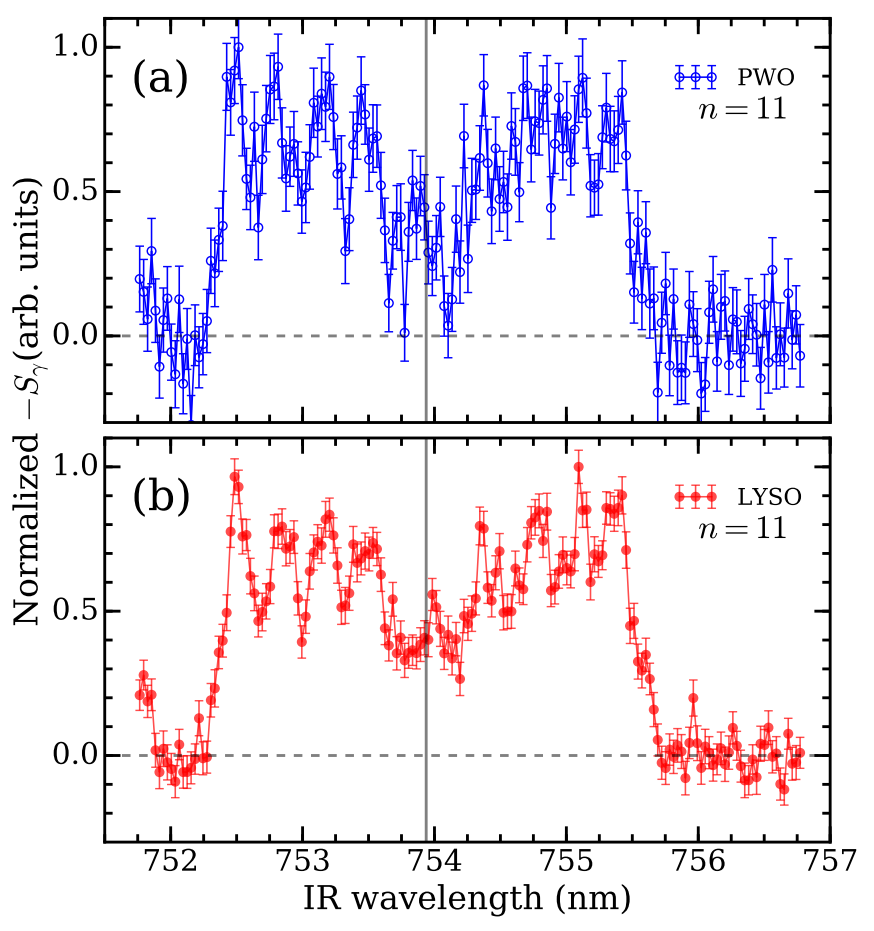

Figure 10: Stark broadened lineshapes for $n=11$ Ps excitation, recorded with (a) PWO and (b) LYSO detectors in an electric field of $1.875 \mathrm{kV} \mathrm{cm}^{-1}$. The acquisition time was 200 seconds per point, or approximately 9 hours for the entire spectra. These data have been normalized to the maximum peak amplitude. The vertical line represents the wavelength of the transition from the $n=2$ gross energy level as described by the Bohr model.

\section{Conclusion}

We have tested a LYSO detector and compared it to PWO for use in SSPALS 9]. We find an enhancement of the SNR in the LYSO case of around a factor of 3, allowing for the acquisition of high quality data. Contrary to expectations, an improved performance was observed when measuring both Ps ionization and the production of Rydberg levels. The advantages of LYSO are attributed entirely to the much higher light output.

We have made a direct comparison to PWO because this was previously thought to be the best material for SSPALS. For experiments that utilize very intense positron pulses, such as studies of molecular positronium [49, PWO is probably still the most appropriate detector material since PMT saturation in these types of experiments is more severe. However LYSO detectors are better suited to the efficient detection of Ps produced from a low-intensity pulsed positron beam. Several groups are currently engaged in such measurements [50, 51, and we anticipate that LYSO will find immediate applications in a number of SSPALS based experiments.

\section{Acknowledgements}

The authors are grateful to L. Liszkay for kindly providing mesoporous silica samples. This work was supported by UCL through its Impact Studentship Programme and was funded in part by the ERC (Grant No. CIG 630119), and the EPSRC (Grant No. EP/K028774/1).

\section{References}

[1] J. R. Danielson, D. H. E. Dubin, R. G. Greaves, and C. M. Surko. Plasma and trap-based techniques for science with positrons. Rev. Mod. Phys. 87, 247 (2015).

[2] A. P. Mills, Jr. and E. M. Gullikson. Solid neon moderator for producing slow positrons. Appl. Phys. Lett. 49, 1121 (1986).

[3] D. B. Cassidy, S. H. M. Deng, R. G. Greaves, T. Maruo, N. Nishiyama, J. B. Snyder, H. K. M. Tanaka, and A. P. Mills, Jr. Experiments with a High-Density Positronium Gas. Phys. Rev. Lett. 95, 195006 (2005).

[4] D. B. Cassidy, K. T. Yokoyama, S. H. M. Deng, D. L. Griscom, H. Miyadera, H. W. K. Tom, C. M. Varma, and A. P. Mills, Jr. Positronium as a probe of transient paramagnetic centers in $\mathrm{SiO}_{2}$. Phys. Rev. B 75, 085415 (2007).

[5] S. Chu and A. P. Mills, Jr. Excitation of the Positronium $1^{3} S_{1} \rightarrow 2^{3} S_{1}$ Two-Photon Transition. Phys. Rev. Lett. 48, 1333 (1982).

[6] K. P. Ziock, R. H. Howell, F. Magnotta, R. A. Failor, and K. M. Jones. First observation of resonant excitation of high- $n$ states in positronium. Phys. Rev. Lett. 64, 2366 (1990).

[7] D. B. Cassidy, T. H. Hisakado, V. E. Meligne, H. W. K. Tom, and A. P. Mills, Jr. Delayed emission of cold positronium from mesoporous materials. Phys. Rev. A 82, 052511 (2010).

[8] A. P. Mills, Jr. and P. Platzman. New Experiments with Bright Positron and Positronium Beams, 115-126. Springer Netherlands (2001).

[9] D. B. Cassidy, S. H. M. Deng, H. K. M. Tanaka, and A. P. Mills, Jr. Single shot positron annihilation lifetime spectroscopy. Appl. Phys. Lett. 88, 194105 (2006).

[10] D. B. Cassidy, S. H. M. Deng, R. G. Greaves, and A. P. Mills, Jr. Accumulator for the production of intense positron pulses. Rev. Sci. Instrum. 77, 073106 (2006).

[11] D. B. Cassidy and A. P. Mills, Jr. A fast detector for singleshot positron annihilation lifetime spectroscopy. Nucl. Instrum. Meth. A 580, 1338 (2007).

[12] A. Rich. Recent experimental advances in positronium research. Rev. Mod. Phys. 53, 127 (1981).

[13] M. Biasini, D. B. Cassidy, S. H. M. Deng, H. K. M. Tanaka, and A. P. Mills, Jr. Suppression of the slow component of scintillation light in $\mathrm{BaF}_{2}$. Nucl. Instrum. Meth. A 553, 550 (2005).

[14] D. B. Cassidy and A. P. Mills, Jr. Interactions Between Positronium Atoms in Porous Silica. Phys. Rev. Lett. 100, 013401 (2008).

[15] D. B. Cassidy, V. E. Meligne, and A. P. Mills, Jr. Production of a Fully Spin-Polarized Ensemble of Positronium Atoms. Phys. Rev. Lett. 104, 173401 (2010).

[16] D. B. Cassidy, P. Crivelli, T. H. Hisakado, L. Liszkay, V. E. Meligne, P. Perez, H. W. K. Tom, and A. P. Mills, Jr. Positronium cooling in porous silica measured via Doppler spectroscopy. Phys. Rev. A 81, 012715 (2010).

[17] A. Deller, B. S. Cooper, T. E. Wall, and D. B. Cassidy. Positronium emission from mesoporous silica studied by laser-enhanced time-of-flight spectroscopy. New J. Phys. 17, 043059 (2015).

[18] D. B. Cassidy, T. H. Hisakado, H. W. K. Tom, and A. P. Mills, Jr. Efficient Production of Rydberg Positronium. Phys. Rev. Lett. 108, 043401 (2012).

[19] A. C. L. Jones, T. H. Hisakado, H. J. Goldman, H. W. K. Tom, A. P. Mills, Jr., and D. B. Cassidy. Doppler-corrected Balmer spectroscopy of Rydberg positronium. Phys. Rev. A 90, 012503 (2014). 
20] T. E. Wall, A. M. Alonso, B. S. Cooper, A. Deller, S. D. Hogan, and D. B. Cassidy. Selective Production of Rydberg-Stark States of Positronium. Phys. Rev. Lett. 114, 173001 (2015).

[21] T. F. Gallagher. Rydberg Atoms. Cambridge University Press (1994).

[22] B. S. Cooper, A. M. Alonso, A. Deller, T. E. Wall, and D. B. Cassidy. A trap-based pulsed positron beam optimised for positronium laser spectroscopy. Rev. Sci. Instrum. 86, 103101 (2015).

23] D. W. Cooke, K. J. McClellan, B. L. Bennett, J. M. Roper, M. T. Whittaker, R. E. Muenchausen, and R. C. Sze. Crystal growth and optical characterization of cerium-doped $\mathrm{Lu}_{1.8} Y_{0.2} \mathrm{SiO}_{5}$. Journal of Applied Physics 88, 7360 (2000).

[24] C. Melcher and J. Schweitzer. A promising new scintillator: cerium-doped lutetium oxyorthosilicate. Nucl. Instrum. Meth. A 314, 212 (1992)

[25] M. E. Phelps. Molecular Imaging with Positron Emission Tomography. Annu. Rev. Nucl. Part. S. 52, 303 (2002).

$26]$ G. Eigen, Z. Zhou, D. Chao, C. Cheng, B. Echenard, K. Flood, D. Hitlin, F. Porter, R. Zhu, G. D. Nardo, C. Sciacca, M. Bizzarri, C. Cecchi, S. Germani, P. Lubrano, E. Manoni, A. Papi, G. Scolieri, A. Rossi, V. Bocci, G. Chiodi, R. Faccini, S. Fiore, E. Furfaro, P. Gauzzi, G. Martellotti, F. Pellegrino, V. Pettinacci, D. Pinci, L. Recchia, A. Zullo, P. Branchini, and A. Budano. A LYSO calorimeter for the SuperB factory. Nucl. Instrum. Meth. A 718, 107 . Proceedings of the 12th Pisa Meeting on Advanced Detectors (2013).

27] I. Valais, S. David, C. Michail, A. Konstantinidis, I. Kandarakis, and G. S. Panayiotakis. Investigation of luminescent properties of LSO:Ce, LYSO:Ce and GSO:Ce crystal scintillators under low-energy -ray excitation used in nuclear imaging. Nucl. Instrum. Meth. A 581, 99 . VCI 2007 Proceedings of the 11th International Vienna Conference on Instrumentation (2007).

[28] B. J. Kemp, C. Kim, J. J. Williams, A. Ganin, and V. J. Lowe. NEMA NU 2-2001 Performance Measurements of an LYSOBased PET/CT System in 2D and 3D Acquisition Modes. J. Nucl. Med. 47, 1960 (2006).

[29] E. Mazzuca, M. Benetti, S. Mariazzi, R. S. Brusa, G. F. D. Betta, and C. Piemonte. Compact gamma detectors based on FBK SiPMs for a Ps Time Of Flight apparatus. J. Instrum. 7, P05006 (2012).

[30] U. Ackermann, W. Egger, P. Sperr, and G. Dollinger. Timeand energy-resolution measurements of $\mathrm{BaF}_{2}, \mathrm{BC}-418$, LYSO and $\mathrm{CeBr}_{3}$ scintillators. Nucl. Instrum. Meth. A 786, 5 (2015).

[31] T. Yamazaki, T. Namba, S. Asai, and T. Kobayashi. Search for $C P$ Violation in Positronium Decay. Phys. Rev. Lett. 104, 083401 (2010).

[32] L. Liszkay, C. Corbel, P. Perez, P. Desgardin, M. F. Barthe, T. Ohdaira, R. Suzuki, P. Crivelli, U. Gendotti, A. Rubbia, M. Etienne, and A. Walcarius. Positronium reemission yield from mesostructured silica films. Appl. Phys. Lett. 92, 063114 (2008)

[33] F. Yang, R. Mao, L. Zhang, and R.-Y. Zhu. Characterization of three LYSO crystal batches. Nucl. Instrum. Meth. A 784, 105. Symposium on Radiation Measurements and Applications 2014 (SORMA XV) (2015).

[34] B. W. Jakoby, D. W. Townsend, M. Sibomana, A. K. LeBlanc, and G. B. Daniel. A Large Volume PET Scanner for Low Dose Applications. Nuclear Science Symposium Conference Record, 2006. IEEE, vol. 5, 3100-3101 (2006).

[35] D. B. Cassidy, T. H. Hisakado, H. W. K. Tom, and A. P. Mills, Jr. Positronium formation via excitonlike states on $\mathrm{Si}$ and $\mathrm{Ge}$ surfaces. Phys. Rev. B 84, 195312 (2011).

[36] P. Crivelli, U. Gendotti, A. Rubbia, L. Liszkay, P. Perez, and C. Corbel. Measurement of the orthopositronium confinement energy in mesoporous thin films. Phys. Rev. A 81, 052703 (2010).

[37] D. B. Cassidy, T. H. Hisakado, H. W. K. Tom, and A. P. Mills, Jr. Laser Excitation of Positronium in the Paschen-Back Regime. Phys. Rev. Lett. 106, 173401 (2011).

[38] B. S. Cooper, A. M. Alonso, A. Deller, L. Liszkay, and D. B.
Cassidy. Positronium production in cryogenic environments. Phys. Rev. B 93, 125305 (2016).

[39] S. M. Curry. Combined Zeeman and Motional Stark Effects in the First Excited State of Positronium. Phys. Rev. A 7, 447 (1973).

[40] A. M. Alonso, B. S. Cooper, A. Deller, S. D. Hogan, and D. B. Cassidy. Positronium decay from $n=2$ states in electric and magnetic fields. Phys. Rev. A 93, 012506 (2016).

[41] H. A. Bethe and E. E. Salpeter. Quantum Mechanics of Oneand Two-Electron Atoms. Springer, Berlin (1957).

[42] P. Coleman. Positron beams and their applications. World Scientific Publishing Co. Pte. Ltd, 1st edn. (2000).

[43] D. B. Cassidy, S. H. M. Deng, and A. P. Mills, Jr. Evidence for positronium molecule formation at a metal surface. Phys. Rev. A 76, 062511 (2007).

[44] E. Vliegen and F. Merkt. Normal-Incidence Electrostatic Rydberg Atom Mirror. Phys. Rev. Lett. 97, 033002 (2006).

[45] S. D. Hogan and F. Merkt. Demonstration of ThreeDimensional Electrostatic Trapping of State-Selected Rydberg Atoms. Phys. Rev. Lett. 100, 043001 (2008).

[46] S. D. Hogan, P. Allmendinger, H. Saßmannshausen, H. Schmutz, and F. Merkt. Surface-Electrode RydbergStark Decelerator. Phys. Rev. Lett. 108, 063008 (2012).

[47] A. P. Mills, Jr. and M. Leventhal. Can we measure the gravitational free fall of cold Rydberg state positronium? Nucl. Instrum. Meth. B 192, 102 (2002).

[48] M. S. Fee, A. P. Mills, Jr., S. Chu, E. D. Shaw, K. Danzmann, R. J. Chichester, and D. M. Zuckerman. Measurement of the positronium $1^{3} S_{1}-2^{3} S_{1}$ interval by continuous-wave two-photon excitation. Phys. Rev. Lett. 70, 1397 (1993).

[49] D. B. Cassidy, T. H. Hisakado, H. W. K. Tom, and A. P. Mills, Jr. Optical Spectroscopy of Molecular Positronium. Phys. Rev. Lett. 108, 133402 (2012).

[50] S. Aghion, C. Amsler, A. Ariga, T. Ariga, A. Belov, G. Bonomi, P. Brunig, J. Bremer, R. Brusa, L. Cabaret, M. Caccia, R. Caravita, F. Castelli, G. Cerchiari, K. Chlouba, S. Cialdi, D. Comparat, G. Consolati, A. Demetrio, L. D. Noto, M. Doser, A. Dudarev, A. Ereditato, C. Evans, J. Fesel, A. Fontana, O. Forslund, S. Gerber, M. Giammarchi, A. Gligorova, S. Gninenko, F. Guatieri, S. Haider, H. Holmestad, T. Huse, I. Jernelv, E. Jordan, T. Kaltenbacher, A. Kellerbauer, M. Kimura, T. Koetting, D. Krasnicky, V. Lagomarsino, P. Lebrun, P. Lansonneur, S. Lehner, J. Liberadzka, C. Malbrunot, S. Mariazzi, L. Marx, V. Matveev, Z. Mazzotta, G. Nebbia, P. Nedelec, M. Oberthaler, N. Pacifico, D. Pagano, L. Penasa, V. Petracek, C. Pistillo, F. Prelz, M. Prevedelli, L. Ravelli, B. Riencker, O. Rhne, S. Rosenberger, A. Rotondi, M. Sacerdoti, H. Sandaker, R. Santoro, P. Scampoli, F. Sorrentino, M. Spacek, J. Storey, I. Strojek, G. Testera, I. Tietje, S. Vamosi, E. Widmann, P. Yzombard, S. Zavatarelli, and J. Zmeskal. Positron bunching and electrostatic transport system for the production and emission of dense positronium clouds into vacuum. Nucl. Instrum. Meth. B 362, 86 (2015).

[51] A. Deller, D. Edwards, T. Mortensen, C. A. Isaac, D. P. van der Werf, H. H. Telle, and M. Charlton. Exciting positronium with a solid-state UV laser: the Doppler-broadened Lyman- $\alpha$ transition. J. Phys. B 48, 175001 (2015). 